\title{
Designing Human-Computer Communication from Epistemic and Cognitive First Principles
}

\author{
Peter Cheng \\ University of Sussex \\ United Kingdom
}

How should we ensure effective communication between humans and digital computing systems? How might visualisations be designed to make transparent deep patterns in complex data? Or interfaces engineered so that users can directly and meaningfully interact with simulation models or computational processes? How could we use AI to adapt the form and sophistication of explanations to suit users with different amounts of target domain knowledge or ability in computational thinking? Current responses to these questions focus upon how information is structured and on the cognitive capabilities of humans. For example, recognition of the potential benefits of graphical interfaces and visualisations are now common place. And designers are increasingly aware of our perceptual, attentional and memory capabilities. This paper goes further by advocating that representations for communication should be designed using epistemic and cognitive first principles. Specifically, effective representations should be created that (a) directly encode the fundamental conceptual structure of their target domain and (b) are compatible with the sophisticated mental processes found in higher forms of cognition, such as problem solving and learning. I will present a selection of past work, from my research group, that takes this approach, including: discovery learning environments for science education; tools for complex problem solving; and visualisations for large quantitative datasets; and also a current project to improve communication between AI systems and humans. 